\title{
The influence of imagery ability on color aftereffects produced by physically present and imagined induction stimuli
}

\author{
JACK BROERSE \\ Mount Gravatt College of Advanced Education, Mount Gravatt 4I22, Australia \\ and \\ BORIS CRASSINI \\ University of Queensland, St. Lucia 4067, Australia
}

\begin{abstract}
Two methods of induction were used to produce orientation-contingent color aftereffects for observers assigned to one of three groups (high, medium, and low) on the basis of self-rated imagery ability. In Experiment 1, observers were required to make magnitude estimates of color aftereffects following inspection of stimulus patterns normally used to produce McCollough effects (e.g., red vertical contours, green horizontal contours). Experiment 2 was a partial replication of Experiment 1, with additional induction conditions in which observers were required to imagine the presence of appropriately oriented contours when particular homogeneous color patches were presented. The results indicated that self-rated imagery ability was not a significant factor in differentiating between observers' performance when orthodox induction procedures were used (Experiment 1). In addition, there were no reliable indications (Experiment 2) that imagined stimulus attributes can be effectively substituted for real stim. ulus attributes in order to produce orientation-contingent color aftereffects. The results are discussed in terms of their implications for the use of imagery-induced perceptual phenomena as a paradigm for investigating the possibility of common neural mechanisms in perception and imagination; in addition, the general implications of the results for understanding the functional significance of self-reported imagery ability are examined.
\end{abstract}

Current empirical investigation of, and theoretical speculation about, the phenomenon of imagery is focused on two closely related issues. The first is concerned with the nature of the mental representations underlying imagery and incorporates the debate between those who argue that "visual imagery is encoded in terms of properties that are quite spatial and modality specific" and others who consider that "imagery is encoded in an abstract propositional format and that this same format is used to encode verbal information" (Anderson, 1978, pp. 249-250; see also Kosslyn \& Pomerantz, 1977). The second issue involves the relationship between perception and imagination; Hebb (1968), for example, proposed that common neural mechanisms are involved in

Final preparation of this manuscript took place while the second author was on Special Studies Program Leave at the Department of Psychology, Pennsylvania State University, and the assistance of members of this department is gratefully acknowledged. Thanks are also due to the principal and staff of the Holland Park State High School and to Sister Mary Murphy and Ian Stephenson for their help in collection of data. Direct reprint requests to either Jack Broerse, Department of Psychology and Special Education, Mount Gravatt College of Advanced Education, Messines Ridge Road, Mount Gravatt 4122, Australia, or to Boris Crassini, Department of Psychology, University of Queensland, St. Lucia 4067, Australia. both the perception of physically present stimuli and the imagination of such stimuli when not physically present. The difference between perception and imagination (according to Hebb, 1968) is that, during the former, higher order neural activity is triggered by sensory input produced by the physical stimuli, while during the latter, higher order activity is triggered by other higher order mechanisms. It is this issue of "functional correspondence" between real and imagined stimuli that forms the basis of the two experiments to be reported.

In broadest terms, the most common paradigm for investigating the relationship between imagination and perception may be characterized as follows: Compare the influence of physically present stimuli on an observer's performance with that of imagined stimuli during the completion of similar tasks. Two distinct types of experiments may be differentiated under this rubric. In the first, the observer is required to respond in some particular manner to stimuli that may either be presented physically or imagined following verbal description. For example, Shepard and Chipman (1970) required observers to judge similarities among the shapes of states of the U.S.A., and it was found that similarity judgments were essentially identical irrespective of whether the states were 
presented pictorially in outline or verbally as state names. From this, Shepard and Chipman (1970) concluded that "our position is consonant with the neuralidentity theory of the mental events ... whatever is going on [when someone is imagining a green square] is functionally similar to what usually goes on when he is confronted with the kind of external object to which we have all associated the words 'green' and 'square"' (p. 16). A somewhat stronger form of this position was recently proposed by Finke and Kosslyn (1980). They compared visual acuity in the periphery, using physically present pairs of dots or dots imagined (after verbal instruction and priming with real dots) as test stimuli. They found similar results for both classes of stimuli and stated that such a result "makes sense if neural mechanisms that are activated when objects are imagined at particular locations in the visual field are like those activated when objects are observed at particular locations in the visual field" (p. 138).

The second type of experiment compares the influence of real and imagined stimuli on an observer's response to some physically present target stimulus. As early as 1910, Perky reported that imagination interfered with perception of real stimuli, in that her observers demonstrated an elevated detection threshold following instructions to imagine particular objects: Despite the presence of real stimuli on every trial, observers reported appropriate stimulus properties in terms of having imagined, rather than of having perceived, the stimuli presented. Segal and her co-workers (Segal \& Fusella, 1970, 1971) replicated and extended the Perky effect using more rigorous experimental methods based on signal detection theory. They reported that the perceptual impairment produced by imagined stimuli is indexed by modality-specific changes in sensitivity $\left(d^{\prime}\right)$, with no concomitant change in response bias $(\beta)$. Podgorny and Shepard (1978) report similar results in a task in which observers were required to make spatial judgments about a physically present probe. The probe appeared in one block of a 5 by 5 block matrix, and observers had to determine whether the block containing the probe had previously formed part of a pattern made up of a number of shaded blocks. Podgorny and Shepard (1978) found no differences in observers' performance, irrespective of whether the shaded pattern was presented pictorially prior to the judgment's being made or was imagined following verbal identification (with or without the outlined matrix as an aid to imagination). The outcome of this study is consistent with the results of the studies reviewed above, which support the notion of a functional equivalence between processes triggered by physical stimuli (perception) and processes lacking external input (imagination). In addition, they support the "pictorial" rather than the propositional models of imagery.
However, the results of other studies have failed to support these notions. Singer and Sheehan (1965) were unable to produce tilt aftereffects following imagination of tilted lines. They demonstrated the classic tilt aftereffect in which a physically present vertical line appears tilted in the direction opposite to that of a previously inspected tilted line (Gibson, 1937). But, after "inspection" of imagined tilted lines, observers reported that the orientation of the vertical target appeared unaltered, or tilted either toward or away from the imaginary tilted lines, depending on the expectations Singer and Sheehan (1965) had produced in their observers through the use of instructions. Singer and Sheehan (1965) discuss these "instruction-dependent" tilt aftereffects in terms of demand characteristics (see also Mitchell \& Richman, 1980; Richman, Mitchell, \& Reznick, 1979). While not exploring the influence of demand characteristics, Over and Broerse (1972) found that imagined vertical lines did not alter the detection threshold of a subsequently presented vertical target, even though the typical angular masking function was found with real masking stimuli. It may be, as Over and Broerse (1972) suggest, that the failure to demonstrate perceptual effects of imagined stimuli in the tilt aftereffect and contour masking experiments is due to the relative simplicity of the imagination task required in these studies; compare, for example, the imagination of tilted or vertical lines with the complex type of visual imagination (trees and dinosaurs) required in the Segal and Fusella (1970) experiment. Although this proposition has some appeal in attempting to account for the discrepant data discussed above, it is unable to explain the recent results of Finke and Kosslyn (1980) or those of Finke and Schmidt $(1977,1978)$ in which imagination of relatively simple stimuli (colors and bars) was found to influence the perceived properties of physical stimuli.

In their first study, Finke and Schmidt (1977) induced orientation-contingent color aftereffects (CAEs), which they claimed were similar to those first reported by McCollough (1965). They instructed their observers either (1) to imagine vertical bars on a physically present, homogeneous patch of green (red) light in alternation with horizontal bars on a physically present green (red) patch, or (2) to imagine green coloration of a physically present vertical (horizontal) grating in alternation with red coloration of a horizontal (vertical) grating. Following imagination of bars, achromatic test gratings were reported as colored in the manner traditionally reported in McCollough effect studies; that is, the CAEs were negative, approximately complementary to the hue paired with the particular grating orientation during imagination. However, after imagination of color, positive CAEs were reported, in which the achromatic gratings appeared the same color as used during 
imagination. In their 1978 study, Finke and Schmidt used a variation of the "imagination of bars" paradigm by presenting observers with spatially ambiguous stimuli on complementary colored backgrounds and instructing them to imagine the ambiguous pattern as either vertical or horizontal bars. Following this adaptation procedure, McColloughtype CAEs were again reported. More importantly, Finke and Schmidt (1978) found that observers with high self-reported imagery ability experienced stronger CAEs than those with low self-reported imagery ability (see also Finke \& Kosslyn, 1980). On the basis of these results, Finke and Schmidt (1978) argued for the "existence of basic perceptuallike associations between color and form, resulting from the projection of imagined form onto physical color fields." In addition, they argued "that color aftereffects established in this manner can serve as a quantitative metric of the individual representation of visual form in images and provide a more sensitive method for inferring the quality of pattern information in imagery, based on the presence of stable, measurable, perceptual-like aftereffects"' (pp. 510, 519; our italics).

Despite the data of Singer and Sheehan (1965) and Over and Broerse (1970), which suggest that tilt aftereffects and orientation masking are uninfluenced by imagined induction stimuli, the results of Finke and Schmidt $(1977,1978)$ seem to indicate that CAEs are not so constrained (leaving aside the issue of the role of demand characteristics in these effects; see later General Discussion). Furthermore, Finke and Schmidt (1978) found that the magnitude of the CAEs induced by imagined stimuli were dependent on the self-reported imagery ability of their subjects. Since Finke and Schmidt argue that the neural processes involved in physical and imagined induction are essentially the same, then it follows that the same relationship between self-reported imagery and magnitude of CAEs reported by Finke and Schmidt for imagined induction should be manifested following induction with physical stimuli. The aim of Experiment 1 was to investigate this expectation.

\section{EXPERIMENT 1}

\section{Method}

Observers. A total of 89 observers (45 females and 44 males) participated: Forty-four students, enrolled in first-year psychology courses at the University of Queensland (satisfying a compulsory course requirement), comprised approximately half the sample, while the remainder were volunteers recruited from psychology staff and students of the Mt. Gravatt College of Advanced Education. All were naive as to the purpose of the experiment, had been screened for normal color vision using Ishihara colorvision test plates, and had normal or corrected-to-normal visual acuity.

Materials and Apparatus. Induction and test stimuli were projected onto a blank white screen, situated at a distance of approximately $2 \mathrm{~m}$ from the observers, ${ }^{1}$ using a Kodak Carousel projector fitted with a $150-\mathrm{W}$ quartz-iodide filament. The spatial characteristics of both induction and test stimuli were identical, consisting of a square wave grating (orientation: horizontal or vertical; spatial frequency: 8 cycles/deg of visual angle) masked to form a circular display (diameter: $12.8 \mathrm{deg}$ of visual angle). Imbedded in this larger circular display was a smaller square wave grating patch (orientation: horizontal or vertical; spatial frequency: 8 cycles $/ \mathrm{deg}$; diameter: $5.8 \mathrm{deg}$ ). The center patch and the surrounding annulus were separated by a distinct black border (width: $18 \mathrm{~min}$ of visual angle). When used as an induction stimulus, the orientation of the square wave pattern in the center and the surround was the same. Kodak Wratten filters were used to produce red (filter No. 26) all-vertical patterns (R-V) or green (filter No. 55) all-horizontal patterns (G-H) for induction purposes. The two achromatic test stimuli contained orthogonally oriented center and surround gratings, that is, a vertical center and a horizontal surround $(\mathrm{V}-\mathrm{H})$ or a horizontal center, and a vertical surround (H-V). The duration of inductionstimulus presentation was controlled by interval generators so that each induction stimulus was alternately presented for $8 \mathrm{sec}$ with an intervening dark interval of $2 \mathrm{sec}$. Exposure durations for test stimuli were similar but under manual control.

During both induction and test phases (see below), the observers sat with their chins on a chinrest and inspected the stimuli through an aperture that enabled the experimenter to occlude vision to either eye, in addition to allowing binocular viewing. Partitions between the observers during group testing ensured that they could not see each other's responses, which were written on score sheets consisting of blank sheets of white paper on which were drawn pairs of concentric circles corresponding to the center/surround configuration of the test stimuli.

Design and Procedure. The observers were tested in groups of not more than four and were seated at separate desks (divided by partitions) forming a single row parallel to the projection screen. As the observers arrived at the laboratory, they were allocated to one of three induction conditions (binocular, monocular left, monocular right). Once they were comfortably seated, with chinrests and viewing apertures suitably adjusted, operation of the viewing aperture was explained in conjunction with the nature of particular induction and test-viewing conditions. The observers were told that following an induction period, during which they were required to inspect the patterns projected onto the screen, test slides would be shown and they would be required to look at these and answer the following questions, in writing, on their score sheets: (1) Did any part of the test slides appear colored? If there was no color, they were to write a zero in the appropriate place on the score sheet. However, (2) if the test slide appeared colored, the subjects were asked to classify the color as either "red" or "green" (forced choice) and, (3) they were asked to estimate the intensity of the color using a scale of 0 to 100 , where 100 indicated a value of color saturation equal to that of the color seen in the induction stimuli. The observers were told that they would be required to make three test judgments: one with both eyes viewing the test stimuli, one with their left eye, and one with their right eye. When the observers understood the various tasks, a pretest period took place in which the two test slides (V-H, H-V) were presented twice in the three test viewing conditions and responses were recorded. The order of test slides was counterbalanced across trials, with the second presentation always being in the reverse order of the first. The same order was maintained from pre- to posttest and was randomized across sessions, with the provision that the first test viewing condition (binocular, monocular left, monocular right) following induction never be identical to the induction viewing condition. Following pretest, the observers inspected alternating R-V and G-H stimuli for an induction period of $10 \mathrm{~min}$, followed by a 30 -sec interval in which they were required to look at the blank screen prior to the commencement of the posttest period (thereby dissipating any spurious afterimages).

After completing induction and test conditions, the imagery questionnaire (Queensland version of the Betts Test of Imagery) was administered. On the basis of scores on this test, the observers 
were allocated to one of three imagery ability groups (high, medium, low). ${ }^{2}$ While one disadvantage of following this particular sequence of testing was an unequal number of observers in some imagery by induction cells, the benefits were, first, that the observers were prevented from forming any preconceptions as to the nature of the task (McCollough effects not normally being formally presented in imagery contexts) and, second, that the experimenters had no prior knowledge of the observers' imagery ability during the measurement of CAEs.

In brief, the design was a three-way mixed factorial with unequal ns. The two between-subjects factors were imagery ability (three levels: high, medium, low) and the induction viewing condition (three levels: binocular, monocular left, monocular right), and the within-subjects factor was the test viewing condition (three levels: binocular, monocular left, monocular right).

\section{Results and Discussion}

As the combination of color and orientation during induction was constant for all subjects (i.e., R-V and G-H), appropriate CAEs were "green" to vertical test stimuli and "red" to horizontal test stimuli. Magnitude estimates of such responses were arbitrarily given plus values. Inappropriate color responses (i.e., "red" to vertical, "green" to horizontal) were given minus values. Responses were standardized by converting each observer's magnitude estimates to proportions of the largest estimate given by the particular observer. ${ }^{3}$ In this way, the maximum pre- or posttest CAE score for someone giving all appropriate responses of equal magnitude was +8 ; the minimum score obtainable with inappropriate responses was -8 . As magnitude estimates for the infrequent number of color judgments obtained during pretest tended to aggregate around zero, pretest scores were subtracted from posttest scores prior to analyses of variance.

To simplify the analysis of results with unequal entries in the imagery ability by induction condition cells, separate two-way, unequal-n analyses of variance were performed on binocular, monocular left, and monocular right induction condition data. Similar patterns were found in all three analyses, indicating that imagery ability did not influence the magnitude of CAEs in any of the induction conditions [binocular induction, $F(2,22)=1.6, p>.05$; monocular left, $F<1.0$; monocular right, $F(2,29)=$ $1.37, \mathrm{p}>.05]$. In addition, the imagery ability factor did not emerge in any significant interactions. The test viewing factor was significant in the analysis of monocular left induction $[\mathrm{F}(2,58)=27.28, \mathrm{p}<.001]$ and monocular right induction data $[F(2,58)=69.94$, $\mathrm{p}<.001]$, but not for binocular induction $[F(2,44)$ $=2.24, \mathrm{p}>.1]$.

As imagery ability did not influence color judgments either as a main effect or in interaction, more detailed analysis of the influence of induction and test viewing conditions was undertaken by collapsing across imagery ability and performing an overall two-way, unequal-n analysis of variance. This revealed a significant main effect for the type of induction $[\mathrm{F}(2,86)=21.79, \mathrm{p}<.001]$, which interacted significantly with test viewing condition $[F(4,172)$ $=37.22, \mathrm{p}<.001]$. The mean CAE scores collapsed over imagery ability are shown in Figure 1 as a function of induction and test conditions. Multiple comparisons (Scheffé, 1953) between particular cell means shown in Figure 1 were undertaken to examine specific properties of the effects produced. Following binocular induction, the CAE magnitudes reported with monocular test viewing did not differ from those produced with binocular test viewing. These CAEs were also similar in magnitude to those produced following monocular induction, but only when the same eye was used during induction and test. For both left and right monocular induction, testing with the ipsilateral eye produced significantly greater effects than testing either binocularly or with the contralateral eye. The magnitudes of CAEs produced with monocular induction and binocular testing were significantly greater than those obtained from testing with the contralateral eye. In fact, neither of the "contralateral" means differed significantly from zero [monocular left induction, monocular right test, $\mathrm{t}(32)=1.31, \mathrm{p}>.05$; monocular right induction, monocular left test, $\mathrm{t}(32)=.4, \mathrm{p}>.05]$.

The results provide evidence consistent with patterns of ocular specificity for CAEs (see Anstis, 1975; Stromeyer, 1978) first reported by McCollough (1965), who was unable to detect any indications that the effect transferred interocularly. ${ }^{4}$ Neural explanations of the lack of interocular transfer have subsequently been based on proposed mechanisms exhibiting dual response properties (color and orientation/spatial selectivity) but receiving input from only one eye (see Over, 1977). Such explanations have received support from neurophysiological inves-

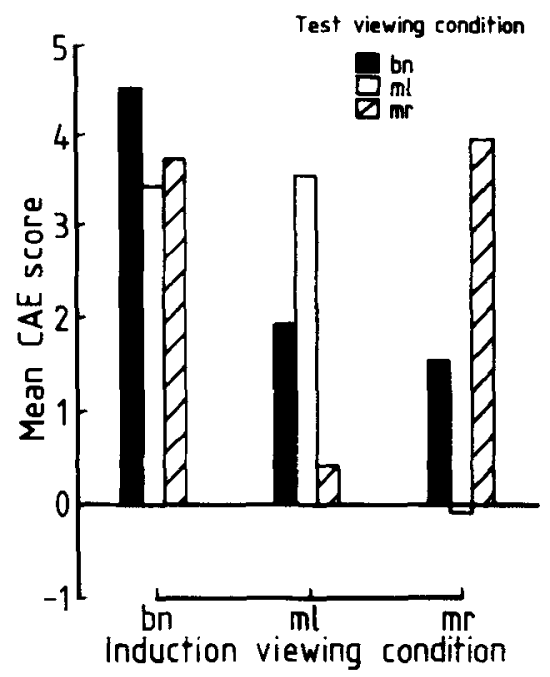

Figure 1. Mean CAE scores as a function of induction and test viewing conditions: For both induction and test, bn = binocular, $\mathbf{m l}=$ monocular left, and $\mathbf{m r}=$ monocular right. 
tigations of single-cell response properties in the infrahuman visual cortex (Gouras, 1974; Hubel \& Wiesel, 1968; Michael, 1978).

Despite the production of quite strong CAEs using normal induction procedures, there was no indication in the data of Experiment 1 that self-reported imagery ability influenced the magnitude of reported CAEs. Observers with low imagery ability as assessed by the Betts test reported CAEs of equal intensity to those reported by observers with high imagery ability. Moreover, both "types" of observers reported CAEs having orientation and ocular specificity normally associated with McCollough effects. Thus, it would seem that, while imagery ability may influence observers' color judgments in the experimental context used by Finke and Schmidt (1978), such influences are not apparent when traditional induction techniques are used. Although there were several procedural differences between Experiment 1 and that of Finke and Schmidt (1978), the crucial difference is that, in the latter, CAE induction took place within an imagery context in which observers were required to use imagery to produce the induction stimuli. If the same neural processes are involved in imagination and perception, this should not matter. However, there are some data (see below) which suggest that this is not the case, and the purpose of Experiment 2 was to compare the relationship between imagery ability and the magnitude of CAEs produced in an imagery context with that found with physical induction.

\section{EXPERIMENT 2}

In Experiment 1, the observers reported the presence or absence of CAEs following the inspection of alternating red vertical and green horizontal bars. No differences in the magnitude of such CAEs were found between groups with self-rated high, medium, and low imagery ability. If self-rated imagery ability indeed reflects differences in underlying neural processes associated with imagery behavior, then the results of Experiment 1 could be taken to indicate that there is no simple and direct link between the neural correlates of imagery phenomena, on the one hand, and the neural correlates of CAEs, on the other. Such interpretations are, however, inconsistent with the finding that subjects who were asked to imagine achromatic bars onto color patches during induction (Finke \& Schmidt, 1977) reported typical negative CAEs during testing. This interpretation is also inconsistent with the finding (Finke \& Schmidt, 1978) that subjects with high self-rated imagery ability consistently reported a greater number of imagery induced CAEs than subjects with low self-rated imagery ability after induction using ambiguous spatial stimuli on color backgrounds.
A possible explanation for such inconsistent reports is that one type of CAE is produced under normal conditions of induction with physical stimuli, while another type (imagery induced CAEs, IICAEs) is produced during induction procedures in which imagery strategies are explicitly involved. Furthermore, it may be that self-reported imagery ability will only influence observers' performance when the latter induction procedures are used. Some support for this possibility is provided by Ashton, McFarland, Walsh, and White (1978), who investigated the influence of self-rated imagery ability on a mental rotation task similar to that used by Cooper and Shepard (1975). Ashton et al. (1978) reported that imagery ability influenced reaction times for making judgments of mental rotation only when subjects were given explicit prior instructions about the use of imagery strategies in the mental rotation task. In the absence of such instructions, there were no differences in reaction times for subjects with high and those with low imagery self-ratings. While these data suggest that imagery ability only becomes a variable of consequence when imagery strategies are explicitly required, comparisons between the results of Ashton et al. (1978) and those of Finke and Schmidt $(1977,1978)$ and Experiment 1 are difficult because of the nature of the task used by Ashton et al. (1978). The aim of Experiment 2, therefore, was to compare the influence of selfreported imagery ability on CAEs produced either through inspection of physical stimuli (a replication of Experiment 1) or imagination of bars on physically present color patches (an extension/replication of Finke \& Schmidt, 1978). In this way, it would be possible to determine whether imagery ability is related only to production of IICAEs, and not CAEs.

\section{Method}

Observers. Thirty-six grade-12 students (aged 16-18 years) at the Holland Park State High School in Brisbane were selected from the total grade-12 population on the basis of their selfratings on the Queensland version of the Betts imagery questionnaire. Twelve students were thus allocated (see Footnote 2 ) to one of three imagery ability groups, as was done in Experiment 1. All observers had normal or corrected-to-normal visual acuity and normal color vision.

Materials and Apparatus. The apparatus and stimuli were the same as those used in Experiment 1. In addition, homogeneous color stimuli were prepared by using the appropriate Kodak Wratten filters (red, No. 26; green, No. 55), masked to produce circular patches of the same overall dimensions as the contoured stimuli used in the present experiment and in Experiment 1.

Design and Procedure. A three-way mixed factorial design was used in which the between-subjects factors were imagery ability (three levels: high, medium, low) and induction viewing condition (two levels: monocular left, monocular right) and the withinsubjects factor was the test viewing condition (two levels: same eye as used for induction, opposite eye of that used for induction). There were equal numbers of males and females in each cell; half the number of observers in each cell viewed the induction stimuli with their left eye, while the remaining half viewed with their right eye. 
The observers were tested in two sessions separated by 1 week. Because of the persistence of McCollough effects induced in the normal manner, the observers were always tested in the imagery conditions first, followed by the condition using physically presented color/contour stimuli 1 week later. The observers were tested in pairs and, once the instructions were clearly understood, they were given a pretest in which the four test slides were viewed, first, with the contralateral eye, and then with the ipsilateral eye. Responses were recorded on score sheets as in Experiment 1. The instructions for the imagery condition (Session 1) were essentially the same as those used by Finke and Schmidt (1977). The observers were first shown achromatic vertical and horizontal gratings and were told they would be required to imagine similar contours whenever the red (imagine vertical) and green (imagine horizontal) color patches were presented. The homogeneous color patches were presented alternately for $8 \mathrm{sec}$ each with a 2 -sec dark interval. After $10 \mathrm{~min}$ of viewing with imagery, the observers looked at the blank screen for $30 \mathrm{sec}$ so that any spurious afterimages would dissipate. Four posttest slides were then presented.

The procedure used in the nonimagery condition (Session 2) was the same as that used in Experiment 1 . In order to eliminate any possible residual effects across sessions, the orientation-color combinations used in Session 1 (R-V, G-H) were reversed for the second session, and the eye used during the second session induction was the opposite of that used in Session 1.

\section{Results and Discussion}

Pre- and posttest color ratings were dealt with as in Experiment 1: Plus values indicate color judgments in the expected direction for normal CAEs and minus values indicate CAE scores in the opposite direction.

The scores were subjected to a three-way analysis of variance, which indicated that imagery ability (high, medium, and low) did not influence magnitude estimates of CAEs in any of the experimental conditions $[F(2,33)<1]$. There were significant main effects for the type of induction procedure $[F(1,33)$ $=88.1, p<.001]$ and for the eye used during the test phase $[\mathrm{F}(1,33)=229.5, \mathrm{p}<.001]$. As indicated in Figure 2, both main effects [interpreted in terms of the significant interaction between them, $F(1,33)$ $=89.3, p<.001]$ indicate clearly that the normal relationship between the ipsilateral and contralateral test eyes found for the interocular transfer of the McCollough effect occurred only in the induction condition in which physically present color/contour stimuli were used. Tests for significance from zero for this induction condition showed that significant appropriate CAEs occurred only when the test stimuli were viewed with the eye used during induction: CAE magnitudes reported using the contralateral eye during testing did not differ significantly from zero. In addition, none of the magnitude estimates of CAEs were influenced by the degree of self-rated imagery ability, thus replicating the results of Experiment 1.

Following induction in which observers were asked to imagine contours, CAE magnitudes of all but one group were not significantly different from zero. That is, few IICAEs were reported. The low imagery-

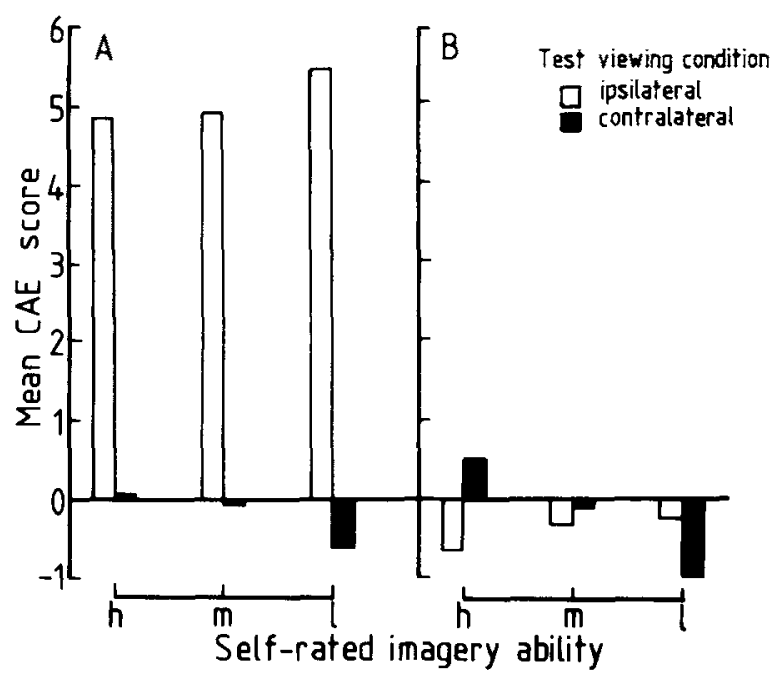

Figure 2. Mean CAE scores as a function of self-rated imagery ability (h $=$ high, $m=$ medium, $1=$ low) and induction and test viewing conditions (ipsilateral $=$ same eye open during induction and test; contralateral = opposite eyes open during induction and test). (A) Induction with physically present color-contour stimuli. (B) Induction with imagined contours.

ability observers reported CAE ratings significantly different from zero $[t(11)=2.15, p<.05]$ when tested with the contralateral eye (see Figure 2). Moreover, the color reports were positive, that is, the same color-orientation relationship as used during induction. None of the other means differed significantly from each other, but the positive effect for the low imagery group was sufficient to produce a significant interaction between imagery ability and the eye used during testing $[\mathrm{F}(1,33)=3.5, \mathrm{p}<.05]$.

Although the low imagery-ability by contralateral test eye cell produced the only evidence (out of six experimental conditions) of color reports following imagery induction (and could thus be considered a chance event), there is some justification at looking at this effect more closely. Finke and Schmidt (1977) also reported positive color aftereffects, but only in the imagery conditions in which the observers were required to imagine appropriate colors when particular contours were presented. In conditions in which colors were imagined to have appropriate contours, as in the present experiment, negative CAEs were reported. Given the relatively small size of the positive effect in the present experiment and the appearance of the positive effect during tests of the contralateral eye only, there would seem to be sufficient doubt about its authenticity as a genuine McCollough-type effect. More importantly, however, the occurrence of "significant" CAEs having orientation/color and ocular specificity unlike the CAEs produced through induction procedures derived from those of McCollough (1965) suggests a need for caution in interpreting these unorthodox CAEs as being similar to normal McCollough ef- 
fects. Before such an interpretation is made, criteria derived from the known properties of McCollough effects (e.g., lack of interocular transfer, dependence on retinal rather than absolute orientation, spatial selectivity) should be included in the design of experiments purporting to demonstrate unorthodox effects, for example, IICAEs.

In summary, the findings of Experiment 2 reinforce the findings obtained in Experiment 1: Under normal induction conditions (physically present contours and colors), appropriate CAEs consistent with the properties of McCollough-type effects in general were observed to the same extent in all three imagery ability groups. However, the imagination of contours on patches of color failed to elicit effects of the type reported by Finke and Schmidt $(1977,1978)$, despite the use of similar imagery instructions.

\section{GENERAL DISCUSSION}

The results of the present experiments address two aspects of the correspondence between imagery and perceptual processes. The first concerns the conjecture that if imagination involves the same neural processes as perception, then imagination of particular stimulus attributes known to produce perceptual phenomena (e.g., aftereffects, threshold elevation, illusions) should result in similar phenomena. Conversely, if similar phenomena are produced through imagination and perception, then common neural mechanisms may be involved. The second aspect concerns the relationship between individual differences in imagery ability as determined by self-report measures and individual differences in performance on perceptual and cognitive tasks in which observers are explicitly instructed, or implicitly assumed, to use imagery as a processing strategy.

\section{Imagery-Induced Effects}

While reliable and appropriate CAEs were produced in all conditions in which normal induction stimuli were used, it was not possible to produce any reliable color reports in those conditions of Experiment 2 in which observers were asked to imagine appropriately oriented contours when presented with real colors. The failure to replicate the effects reported by Finke and Schmidt $(1977,1978)$ under conditions of imagery induction may simply indicate that the measurement procedures in the present experiment were too stringent. Finke and Schmidt (1977, 1978) used a forced-choice procedure that did not permit a response of "no color." Their subjects were asked to indicate which of two simultaneously presented orthogonal gratings appeared most red or least green. Furthermore, Finke and Schmidt (1977, 1978) did not use a pretest/posttest design, which allows for a correction for any response bias in the subjects' color responses. Given that subjective col- ors similar in some respects to McCollough effects may be reported in the absence of induction (see Stanley \& Hoffman, 1976), the use of a pretest procedure seems essential to ensure that reported CAEs are the result of the induction process, whatever its form, and not of other factors unrelated to the induction process (e.g., subjects' expectations or experimenters' demand characteristics; see Mitchell \& Richman, 1980; Richman et al., 1979).

An additional feature of the Finke and Schmidt $(1977,1978)$ studies was the use of postexperimental interrogation of subjects to determine response strategies. On the basis of these interrogations, the data of approximately half the subjects in both studies were discounted because subjects reported using associative learning and memory strategies during testing. In these cases, subjects associated a particular color with a particular orientation during induction so that, during testing, the same color was reported with its "associated" orientation. Despite the rationale given by Finke and Schmidt (1978, see pp. 518, 520), it seems an unnecessarily ad hoc method of culling data. A similar end may be achieved in a less questionable manner by incorporating checks for interocular transfer in the design, as was the case in Experiment 2. Observers using associative learning strategies are likely to give positive rather than negative CAEs and to report such CAEs when tested with even the contralateral eye when no CAEs are expected. Presenting test patterns to the contralateral eye is thus analogous to presenting "catch" trials, albeit on an informal basis. In this way, color reports can be shown to be consistent or inconsistent with CAEs produced in the majority of investigations of properties of McCollough effects.

This point needs to be stressed. The argument that the same neural processes are involved in imagination and perception is critically dependent upon the assumption that CAEs, for example, produced by imagination and perception are the same. The data of Experiment 2, and indeed the data of Finke and Schmidt $(1977,1978)$, suggest that this is not the case. It seems that IICAEs (1) are dependent on whether subjects imagine bars on physically present color patches or color on physically present bars, (2) are susceptible to different associative/cognitive strategies, and (3) are much weaker than CAEs produced normally. These factors indicate that the IICAEs reported by Finke and Schmidt $(1977,1978)$ are not like normal CAEs, and that conclusions based on a premise of identity between the two may be in error.

\section{Imagery Ability}

The data of Experiment 1 indicated that when induction procedures involving real colors and real contours are used to generate CAEs, there were no 
individual differences in the performance of observers on the basis of previously measured imagery ability. These results were replicated in Experiment 2 in those conditions in which similar induction procedures were used. While such results are disappointing from the point of view of the hypothesis of imagery/ perception involving common neural mechanisms, they are surprisingly consistent with a number of studies investigating the functional significance of imagery as measured by self-report questionnaires. Recent surveys of this literature (e.g., Ernest, 1977; White, Sheehan, \& Ashton, 1977) have indicated some considerable difficulties in broaching the question of construct validity of such questionnaire-assessed imagery ability. Thus, while measured imagery ability continues to be functionally linked to specific performance indices (e.g., reaction time) of perceptual and cognitive phenomena (e.g., mental rotation, recognition and recall tasks), an understanding of the nature of the processes underlying these links remains relatively obscure. In particular, such findings say relatively little about conjectures of neural substrates common to imagery, perception, and cognition.

In recent years, however, certain classes of perceptual phenomena (e.g., threshold elevation, spatial aftereffects) have been rather successfully explained in terms of some basic neural processes (see Results and Discussion, Experiment 1). Similar neural mechanisms have been directly implicated as a neural prototype for imagery (Fink \& Kosslyn, 1980; Podgorny \& Shepard, 1978) so that the potential benefits of establishing imagery-related individual differences on such perceptual tasks are obvious. In this regard, further studies of the McCollough effect are even more compelling in that they have also been linked with other cognitive processes, for example, learning and memory (see Shute, 1979). The failure, in the present study, to identify such individual differences might well reinforce some recent claims that subjective self-report measures of imagery ability are substantially independent of objective performance in tasks requiring the use of imagery processes (e.g., White et al., 1977).

\section{Conclusion}

In their recent discussions of the relationship between self-report imagery assessment schedules and objective performance indices of imagery processes, both Ernest (1977) and White et al. (1977) argue the need for increased investigation of the nature of imagery processes, rather than merely demonstrating a functional equivalence between performance factors and self-reported imagery ability. Imagery-induced perceptual phenomena (e.g., aftereffects, threshold elevation effects) may provide potentially useful paradigms to meet such demands. However, before such phenomena are interpreted as indicating common neural mechanisms between imagination and per- ception, it is necessary to determine their reliability. In addition, it must be ensured that imagery-induced "perceptual" phenomena display the same characteristics as the physically induced "perceptual" phenomena they are supposed to mimic. On the basis of the present data, IICAEs appear to fail on both counts.

\section{REFERENCES}

Anderson, J. R. Arguments concerning representations for mental imagery. Psychological Review, 1978, 85, 249-277.

Anstis, S. M. What does visual perception tell us about visual coding? In M. S. Gazzaniga \& C. Blakemore (Eds.), Hondbook of psychobiology. New York: Academic Press, 1975.

Ashton, R., McF ARLAND, K., WALSh, F., \& White, K. Imagery ability and the identification of hands: A chronometric analysis. Acta Psychologica, 1978, 42, 253-262.

Cooper, L. A., \& Shepard, R. N. Mental transformations in the identification of left and right hands. Journal of Experimental Psychology: Human Perception and Performance, 1975 , 1, 48-56.

Ernest, E. I. Imagery ability and cognition: A critical review. Journal of Mental Imagery, 1977, 2, 181-216.

Finke, R. A., \& Kossi.yn, S. M. Mental imagery acuity in the peripheral visual field. Journal of Experimental Psychology: Human Perception and Performance, 1980, 6, 126-139.

Finke, R. A., \& SchmidT, M. J. Orientation-specific color aftereffects following imagination. Journal of Experimental Psy. chology: Human Perception and Performance, 1977, 3, 599-606.

Finke, R. A., \& SchmidT, M. J. The quantitative measure of pattern representation in images using orientation-specific color aftereffects. Perception \& Psychophysics, 1978, 23, 515-520.

Gibson, J. J. Adaptation, aftereffect, and contrast in the perception of tilted lines. II. Simultaneous control and the areal restriction of the aftereffect. Journal of Experimental Psychology, 1937, 20, 553-569.

Gouras, P. Opponent-colour cells in different layers of foveal striate cortex. Journal of Physiology, 1974, 238, 583-602.

Hквв, P. O. Concerning imagery. Psychological Review, 1968, $75,466-477$

Hubel, D. H., \& Wiesel, T. N. Receptive fields and functional architecture of monkey striate cortex. Journal of Physiology, $1968,195,215-243$.

Kosslyn, S. M., \& Pomerantz, J. R. Imagery, propositions, and the form of internal representations. Cognitive Psychology, $1977,9,52-76$.

Mackay, D. M., \& MaCKay, V. Orientation-sensitive after-effects of dichoptically presented colour and form. Nature, 1973, 242, 477-479.

McCollough, C. Color adaptation of edge detectors in the human visual system. Science, 1965, 149, 1115-1116.

MichaEl, C. R. Color vision mechanisms in monkey striate cortex: Simple cells with dual opponent color receptive fields. Journal of Neurophysiology, 1978, 41, 1233-1249.

Mitchell, D. B., \& Richman, C. L. Confirmed reservations: Mental travel. Journal of Experimental Psychology: Human Perception and Performance, 1980, 6, 58-66.

Oven, R. Contingent aftereffects in the perception of colour and contour. In R. H. Day \& G. V. Stanley (Eds.), Studies in perception. Perth: University of Western Australia Press, 1977.

Over, R., \& Broerse, J. Imagined lines fail to induce contour masking. Psychonomic Science, 1972, 29, 203-204.

Podgorny, P.. \& ShePard, R. N. Functional representations common to visual perception and imagination. Journal of Experimental Psychology: Human Perception and Performance, $1978,4,21-35$

PotTs, M. J. \& Harris, J. P. Dichoptic induction of movement aftereffects contingent on color and on orientation. Perception \& Psuchophysics, 1979. 26. 25-31 
Richman, C. L., Mitchell, D. B., \& Reznick, J. S. Mental travel: Some reservations. Journal of Experimental Psychology: Human Perception and Performance, 1979, 5, 13-18.

Scheffé, H. A. A method for judging all contrasts in the analysis of variance. Biometrika, 1953, 40, 87-104.

Segal, S. J., \& Fusella, V. Influence of imaged pictures and sounds on detection of visual and auditory signals. Journal of Experimental Psychology, 1970, 83, 458-464.

Segal, S. J., \& Fusella, V. Effect of images in six sense modalities on detection of visual signal from noise. Psychonomic Science, 1971, 24, 55-56.

ShFPARD, R. N., \& Chipman, S. Second order isomorphism of internal representations: Shapes of states. Cognitive Psychology, $1970,1,1-17$.

Shute, C. C. D. The McCollough effect. Cambridge, England: Cambridge University Press, 1979.

Singer, G., \& Sheman, P. W. The effect of demand characteristics on the figural aftereffect with real and imaged inducing figures. American Journal of Psychology, 1965, 78, 96-101.

Stantey, G., \& Hofrman, W. C. Orientation-specific color effects without adaptation. Bulletin of the Psychonomic Society, 1976, 7, 513-514.

Stromeyer, C. F., III. Form-color aftereffects in human vision. In R. Held, H. Leibowitz, \& H.-L. Teuber (Eds.), Handbook of sensory physiology (Vol. 8): Perception. Heidelberg: SpringerVerlag, 1978.

VidyasagaR, T. R. Orientation specific colour adaptation at a binocular site. Nature, 1976, 261, 39-40.

White, K., Sheehan, P. W., \& Ashton, R. Imagery assessment: A survey of self report measures. Journal of Mental Imagery, 1977, 1, 145-170.

White, K. D., Petry, H. M., Riggs, L. A., \& Miller, J. Binocular interactions during establishment of McCollough effects. Vision Research, 1978, 18, 1201-1215.

\section{NOTES}

1. It was possible to test four observers simultaneously, two being seated on either side of the projector. The viewing distance of the two observers nearest the projector was $2 \mathrm{~m}$, while that of the other two observers was $2.12 \mathrm{~m}$. The spatial characteristics of the stimuli are expressed in terms of the 2-m viewing distance.

2. The range of scores on the Queensland version of the Betts test is 35 (perfectly vivid imagery) to 245 (no imagery ability). In Experiment 1, the criteria for high, medium, and low imagery ability were Betts scores of 90 or less (mean $=70, S D=15, n=29$ ), Betts scores between 91 and 110 (mean $=102, S D=7, n=28$ ), and Betts scores of 111 or greater (mean $=140, S D=20, n=32$ ), respectively. In Experiment 2, observers were preselected and allocated to equal-sized imagery ability groups to conform to the imagery ability criteria used in Experiment 1.

3. Observers varied greatly in their magnitude estimations of the most saturated CAE, estimates ranging from .5 to 90 on the allowable 0-100 scale. Analysis of those estimates indicated no systematic relationship between self-rated imagery ability and maximum magnitude estimation. The standardization procedure used to derive CAE scores served to minimize variability produced by individuals' choices of maximum magnitude estimates.

4. There have been some reports of dichoptic interactions in the production of CAEs; however, these reports involve atypical induction procedures. For example, Mackay and Mackay (1973) presented homogeneous colored fields to one eye and achromatic gratings simultaneously to the other. Observers reported normal CAEs when tested with the eye stimulated by colored fields and positive CAEs (in which the color-orientation relationship is the opposite of normal CAEs) when tested with the other eye. Vidyasagar (1976) used an even more complex induction procedure with binocular viewing of induction stimuli alternated with monocular viewing of induction stimuli designed to "cancel" the binocular CAEs. Observers reported normal CAEs when tested binocularly and positive CAEs when tested monocularly. While these dichoptic interactions are of interest in investigations of the neural basis of CAEs (e.g., see Potts \& Harris, 1979; Stromeyer, 1978; White, Petry, Riggs, \& Miller, 1978), they are not relevant to the present experiments because of particular induction procedures adopted.
(Received for publication April 7, 1980; revision accepted October 22,1980 .) 